\title{
The effect of a color tattoo on the local skin redox regulatory network: an $\mathrm{N}$-of- 1 study
}

\author{
Jan Homolak ${ }^{1,2}$ \\ ${ }^{1}$ Department of Pharmacology, University of Zagreb School of Medicine, Zagreb, \\ Croatia \\ ${ }^{2}$ Croatian Institute for Brain Research, University of Zagreb School of Medicine, \\ Zagreb, Croatia
}

\begin{abstract}
Introduction:

Tattooing has been extensively discussed in the literature in the context of hygiene and infectious diseases. On the other hand, pathophysiological effects of tattoo inks in the human body are yet to be elucidated. Oxidative stress is considered to be the most important mediator of potential adverse effects of tattooing, however at the moment there is no experimental evidence for tattoo ink-related oxidative stress in the human body. The aim of this study was to examine the effect of a blue tattoo on skin redox regulatory network (RRN) parameters in a single human subject.
\end{abstract}

\section{Materials and Methods:}

A blue tattoo on my left forearm was used in the research. Tattooed skin surface oxidationreduction potential (ORP) experiment was done by a PH60F flat probe. Capillary blood from the tattoo and the control area was extracted and analyzed with $\mathrm{I}_{2} / \mathrm{KI}$-stabilized microORP, nitrocellulose redox permanganometry (NRP), carbonato-cobaltate (III) formation-derived $\mathrm{H}_{2} \mathrm{O}_{2}$ dissociation rate assay, 1,2,3-Trihydroxybenzene autoxidation assay, thiobarbituric reactive substances (TBARS) assay and 5,5,'-dithio-bis-(2-nitrobenzoic acid) (DTNB)-based determination of free thiol content in low molecular weight and protein precipitate fraction.

\section{Results:}

Surface ORP analysis revealed a greater antioxidant capacity of tattooed skin in comparison with the control (CTR). Capillary blood analysis confirmed slightly greater reductive capacity in the tattoo sample both by microORP $(-4.33 \mathrm{mV}$ vs CTR) and NRP $(+1.1 \%)$. Hydrogen peroxide dissociation rate $(+11.8 \%)$, superoxide dismutase activity $(+0.7 \%)$, and protein sulfhydryl content $(+8.5 \%)$ were all increased, and lipid peroxidation $(-15 \%)$ and low molecular weight thiols $(-2.8 \%)$ were reduced in a tattoo sample in comparison with the CTR.

\section{Conclusion:}

In this N-of- 1 study RRN of tattooed skin was shifted towards a more reductive state with most of the parameters indicating reduced levels of oxidative stress in comparison with nontattooed skin. Local antioxidant effect of copper(II) phthalocyanine provides one possible explanation of the observed effects.

Keywords: Tattoo, Tattoo ink, Oxidative stress, Phthalocyanine, Skin 
Conflict of interest statement: I have no conflict of interest to disclose.

Funding source: None

\section{Introduction:}

Tattooing, a practice of inserting ink, dyes and pigments into the dermis for aesthetic reasons is becoming increasingly popular especially among young people. Medical consequences of tattooing have been thoroughly discussed in the literature, however mostly in the context of hygiene and transmission of infectious diseases. On the other hand, pathophysiological consequences of tattooing are still largely unknown. A cursory look at the chemical constituents of tattoo inks reveals a great chemical variability with an equally enormous list of possible biological effects of different chemicals and their combinations. Composition and toxicological effects of tattoo inks have been reviewed in detail in the literature and interested readers are pointed to informative articles by Arl et al. (Arl et al. 2019) and Laux et al. (Laux et al. 2016). The main components of tattoo inks are usually divided into several categories with the most important being vehicles (eg. water and glycerine), additives (eg. surfactants, polycyclic aromatic hydrocarbons, nanoparticles and polymers), pigments (polycyclic compounds) and metals (eg. titanium, barium, aluminium and copper as colourants, or arsenic, cadmium, chromium, cobalt, lead or nickel as contaminants)(Arl et al. 2019; Forte et al. 2009a; Forte et al. 2009b; Laux et al. 2016; Serup et al. 2020). Black and coloured inks differ greatly in their chemical composition. Black ink often contains iron(II) oxide (FeO) and carbon, red is often based on cadmium selenide (CdSe) also known as cadmium red, green ink contains chromium(III) oxide $\left(\mathrm{Cr}_{2} \mathrm{O}_{3}\right)$, and blue cobalt(II) aluminate $\left(\mathrm{CoAl}_{2} \mathrm{O}_{4}\right)$ (Athar and Farrukh 2019). Additionally, modern red and yellow tattoo inks contain azo dyes, and phthalocyanines are usually found in different shades of blue and green (Bäumler et al. 2000). Significant chemical variability of tattoo inks is probably responsible for different biological effects. For example, one in vitro study on cellular viability and protein synthesis in human fibroblasts exposed to different tattoo inks reported that there was no toxic effect of commonly used black ink Strong Black, however red ink Biolip 27 significantly reduced viability and decreased expression of procollagen a1 type I protein (Falconi et al. 2009). One other study tested 19 different black tattoo inks and found significant variability in both their chemical constitution and the potential to reduce mitochondrial activity of human keratinocytes in vitro (Regensburger et al. 2010). Apart from great chemical variability and complexity of commonly used tattoo inks, another challenge for understanding pathophysiological effects of tattooing arises from the fact that tattooing has never been extensively studied in humans in vivo. Biological differences of animals, cells and humans are well known in pharmacology where many drugs display promising effects in vitro and in laboratory animals, but fail to induce biological effects in humans, or 
even worse detrimental effects manifest for the first time in human trials due to significant biochemical, physiological and structural differences. However, the majority of studies on the toxic effects of tattoo inks were done in different cell lines in vitro, and papers describing biological effects of tattoos in humans are extremely scarce. Moreover, not a single study I am aware of examined the effect of tattoo ink on oxidative stress in humans.

A lucky coincidence that I am actively involved in redox regulation research and prone to self-experimentation in combination with the fact that I was equipped with adequate curiosity and a blue tattoo on my left forearm motivated me to conduct an N-of- 1 study on myself to try to understand how the presence of chemical constituents of a color tattoo affects local skin redox regulatory network.

\section{Materials and methods:}

\section{Skin oxidation-reduction potential (ORP)}

Skin was thoroughly cleaned with soap, water and distilled water and dried. Surface skin ORP measurements were conducted with PH60F (Apera Instruments, Germany) with a flat end to ensure optimal contact of the electrode with the skin and the instrument was calibrated and cleaned prior to measurements. A tattoo area was divided in 12 equal parts as shown in Fig 1B. Measurements were designed in a way to maximally reduce experimental error. Every area was measured once, followed by the corresponding control area on the forearm without a tattoo (1-tattoo, 1-control, 2-tattoo, 2-control,...) and everything was repeated three times. A mean value of three measurements was used even though variations were minimal. To exclude possible unilateral differences due to other physiological reasons additional body parts were chosen as controls for unilateral variation - skin above the 5th rib in the anterior axillary line ( $5^{\text {th }} \mathrm{RIB} \mathrm{AAL)}$ and clavicular (anterior) part of the deltoid muscle (SHOULDER).

\section{Prick sampling and sample preparation}

In order to explore local RRN capillary blood was drawn from two locations on the tattooed forearm as shown in Fig 1A. Skin was cleaned as described previously, and a lancing device was used to obtain a local blood sample with a new lancet used each time. One microliter of the blood was taken from each site with a micropipette and dissolved in $39 \mu \mathrm{l}$ of double distilled water $\left(\mathrm{ddH}_{2} \mathrm{O}\right)$ validated for $0.055 \mu \mathrm{S} / \mathrm{cm}$. Samples were vortexed thoroughly and stored at $20^{\circ} \mathrm{C}$.

\section{Sample oxidation-reduction potential}

In vitro sample reductive capacity was measured by means of $\mathrm{I}_{2} / \mathrm{KI}$-redox-coupled microORP to ensure redox system stabilization as described previously (Cao et al. 2016). In short, $0.1 \mathrm{M}$ $\mathrm{I}_{2}$ and $0.4 \mathrm{M} \mathrm{KI}$ solutions were used as the redox pair electrode solution in the experiment. Samples $(2 \mu \mathrm{l})$ were mixed with $5 \mu \mathrm{l}$ of redox solution and left for 1 hour in the dark at $25^{\circ} \mathrm{C}$. After the incubation period, samples were measured in three time points with redox microsensor system ORP-146S (Shelf scientific, Lazar Research Laboratories, Inc., Los Angeles, USA) composed of a platinum sensing element with $\mathrm{Ag} / \mathrm{AgCl}$ reference $(\mathrm{KCl}$ was used as a 
filling solution). Readings were obtained by $6230 \mathrm{~N}$ Microprocessor meter (Jenco Instruments, San Diego, USA). System accuracy was $\pm 0.5 \mathrm{mV}$. Readings were taken as $\mathrm{mV}$ differences between the control and the tattoo sample.

\section{Nitrocellulose Redox Permanganometry (NRP)}

Total reductive capacity of the sample was additionally checked with NRP to control for reductive specificity and overcome possible bias due to a redox pair used for ORP stabilization. A Standard NRP protocol was used (Homolak et al. 2020a). Briefly, $1 \mu$ l of each sample was placed on a nitrocellulose membrane (Amersham Protran 0.45; GE Healthcare Life Sciences, USA), left to dry out, and developed in $\mathrm{KMnO}_{4}$ solution $\left(0.2 \mathrm{~g} \mathrm{KMnO}_{4}\right.$ in $20 \mathrm{ml}$ $\mathrm{dd} \mathrm{H}_{2} \mathrm{O}$ ). Excess reagent was removed under flowing $\mathrm{dH}_{2} \mathrm{O}$, and $\mathrm{MnO}_{2}$ precipitate was visualized by scanning. Membrane was analyzed in Fiji (NIH, USA) by means of GelAnalyzer plugin for integrated density quantification.

\section{Hydrogen peroxide dissociation rate}

Hydrogen peroxide dissociation rate indicating the activity of peroxidases was done by multipoint $\mathrm{H}_{2} \mathrm{O}_{2}$ quantification derived from carbonato-cobaltate(III) complex ([Co(CO3)3]Co) spectrophotometric assessment (Hadwan 2018). In short, samples were analyzed multiple times until concentration with corresponding activities was inside the quantification range. Once optimal dilution was determined, samples were incubated with $40 \mu \mathrm{l} 10 \mathrm{mM} \mathrm{H}_{2} \mathrm{O}_{2}$ dissolved in phosphate buffered saline for 60 seconds, and the reaction was stopped by adding $100 \mu \mathrm{L}$ of cobalt(II) hexametaphosphate working solution. Absorbance of the carbonato-cobaltate complex was determined at $450 \mathrm{~nm}$ with Infinite F200 PRO multimodal microplate reader (Tecan, Switzerland).

\section{Superoxide dismutase}

Superoxide dismutase activity was assessed by 1,2,3-Trihydroxybenzene autooxidation inhibition determined from quantification of absorbance increment at $325 \mathrm{~nm}$ for 300 seconds(Li 2012). Briefly, $10 \mu \mathrm{l}$ of the sample was added to $1000 \mu \mathrm{l}$ of $0,05 \mathrm{M}$ Tris- $\mathrm{HCl}$ and $1 \mathrm{mM} \mathrm{Na} \mathrm{N}_{2}$ EDTA ( $\mathrm{pH} 8,2$ ) and vortexed in the eppendorf tube. $15 \mu \mathrm{L}$ of $60 \mathrm{mM}$ 1,2,3trihydroxybenzene dissolved in $1 \mathrm{mM} \mathrm{HCl}$ was added and the sample was briefly vortexed again and pipetted into the spectrophotometric cuvette. Absorbance difference was analyzed and compared to the increment of the standard sample. CamSpec M350 DoubleBeam UVVisible Spectrophotometer (Cambridge, UK) was used in the experiment.

\section{Thiobarbituric reactive substances assay (TBARS)}

TBARS assay was used for assessment of lipid peroxidation(Prabhakar et al. 2012). In short, $10 \mu \mathrm{L}$ of the sample was mixed with $190 \mu \mathrm{L}$ of $\mathrm{ddH}_{2} \mathrm{O}$ and $400 \mu \mathrm{L}$ of TBARS reagent $(0,375 \%$ thiobarbituric acid; $15 \%$ trichloroacetic acid). Samples were incubated at $95^{\circ} \mathrm{C}$ for 30 minutes in perforated eppendorf tubes and placed under tap water to cool down afterwards. Once the sample was cool, $600 \mu \mathrm{L}$ of $\mathrm{n}$-butanol was added to the mixture to extract malondialdehydethiobarbituric acid (MDA-TBA) chromogen. Absorbance of the butanol fraction was analyzed at $532 \mathrm{~nm}$ and the amount of TBARS was estimated based on the molar extinction coefficient 
of $1,56 \times 10^{5} \mathrm{M}^{-1} \mathrm{~cm}^{-1}$. CamSpec M350 DoubleBeam UV-Visible Spectrophotometer (Cambridge, UK) was used in the experiment.

\section{Protein sulfhydryl content and low molecular weight thiol determination}

Protein thiols and low molecular weight thiols (LMWT) were determined by reacting the samples with 5,5'-dithio-bis(2-nitrobenzoic acid) (DTNB) with subsequent quantification of 5thio-2-nitrobenzoic acid (TNB)(Prabhakar et al. 2012; Van der Plancken et al. 2005). Briefly, samples $(10 \mu \mathrm{L})$ were mixed with $15 \mu \mathrm{L}$ of $\mathrm{dd}_{2} \mathrm{O}$ and $25 \mu \mathrm{L}$ of sulfosalicylic acid (4\% w/v) was added to the mixture. Samples were left on ice in dark for 1 hour and spun at 10000 RPM for 10 minutes afterwards. Supernatant $(45 \mu \mathrm{L})$ was reacted with $45 \mu \mathrm{L}$ of DTNB reagent $(4 \mathrm{mg} / \mathrm{ml}$ in $5 \%$ sodium citrate) for analysis of LMWT, and remaining solution was removed from the tubes by gentle tapping and $45 \mu \mathrm{L}$ was reacted with the protein precipitate. After the reaction, samples were placed in a transparent 96 well plate and analyzed with F200 PRO multimodal microplate reader (Tecan, Switzerland) at $405 \mathrm{~nm}$. Protein precipitate was analyzed with the addition of potassium phosphate buffer $(0,1 \mathrm{M}, \mathrm{pH} 7,4)$ to increase the volume.

\section{Results:}

\section{Skin surface ORP}

Analysis of skin surface ORP revealed that tattooed skin had increased reductive potential in comparison to the surface of the control skin area. In other words, slightly lower ORP values suggest that tattooed skin was less oxidative. This finding was at first counterintuitive as literature suggests increased oxidative stress in tattooed skin, however, out of 72 consecutive measurements ( 36 of the 12 tattoo areas of interest and 36 of the 12 control areas as explained in the Methods section) 54 measurements showed that tattooed skin was stronger antioxidant, 12 measurements suggested it had the same reductive potential as the control skin, and only 6 measurements suggested tattooed skin was slightly more oxidative. Measurement areas are presented in Fig 1B and mean results of all areas are shown in Fig 1C. Control measurements are presented in Fig 1D.

\section{Skin capillary blood RRN}

As surface skin measurements suggested tattooed skin was less oxidative, I decided to obtain capillary blood samples from tattooed and control skin to assess oxidative stress parameters from the local extracellular pool that should reflect redox status of the surrounding biological environment. Here, I analyzed ORP in vitro with a redox micro measurement system as described in the Methods section and obtained similar results as tattoo samples were again few $\mathrm{mV}$ more reductive in comparison with the control samples (Fig 1E). Furthermore, samples were analyzed by NRP, a novel method characterized by great accuracy and precision for measurement of reductive capacity in tiny volumes of biological samples (Homolak et al. 2020a). Nitrocellulose redox permanganometry confirmed both surface measurements and in vitro ORP and indicated increased antioxidant capacity of the tattooed skin (Fig 1F). As both methods used for overall redox balance analysis indicated that a small antioxidative shift was present in the tattoo samples (Table 1), I decided to analyze specific subsystems involved in cellular redox regulation to see what mechanisms were responsible for the observed effect. As shown in Fig 1G, hydrogen peroxide dissociation rate was 
increased in tattoo samples by $11.8 \%$ indicating greater activity of catalase and other peroxidases in the inked skin. Superoxide dismutase activity was also increased, however only for $0.7 \%$ in comparison with the control (Fig 1H). Lipid peroxidation measurement was the most surprising as tattoo samples had $15 \%$ less harmful electrophile aldehyde peroxidation end products as determined by the TBARS assay (Fig 1I). Protein sulfhydryl content was also slightly increased in tattoo samples (+8.5\%; Fig 1J) indicating less proteins were oxidised by environmental redox homeostatic perturbations. Low molecular weight thiols were the only RRN subsystem that suggested tattooed skin was exposed to more oxidative stress in comparison to non-tattooed skin, however the results suggest a very small reduction of only $2.8 \%$ of the control values (Fig $\mathbf{1 K}$ ).

\section{Discussion:}

Taken together, the results of this experiment strongly suggest that oxidative stress was reduced, rather than increased in the tattooed skin in this particular case. I personally find this extremely interesting as I originally expected to see the exact opposite. As briefly mentioned in the introduction, biological phenomena underlying tattoo-induced changes are still largely unexplored. Nevertheless, oxidative stress has been proposed as the most important pathophysiological mediator responsible for tattoo-related health complaints such as swelling, itching and redness following solar radiation exposure in the famous "Beach study" (Hutton Carlsen and Serup 2014). Interestingly, association of reactive oxygen species (ROS) and tattoo ink in humans has so far only been supported by indirect findings from in vitro toxicological studies (Høgsberg et al. 2013; Neale et al. 2015) and theoretical assumptions based on the fact that mechanisms of photoactivation underlying the biological effects of photodynamic therapy were also shown for some chemicals that are often present in tattoo inks such as polycyclic aromatic hydrocarbons (Hutton Carlsen and Serup 2014; Srivastav et al. 2020). Nevertheless, PubMed search for "tattoo" AND "oxidative stress" and "tattoo" AND "ROS" only yield 7 and 8 hits respectively, and none of the results provide any evidence for direct oxidative stress effects of tattooing in humans. Consequently, this N-of-1 study, although limited by its design, provides first reported evidence of redox system-related effects of tattoo ink in the human body. Interestingly, the results seem to contradict the hypothesis that tattoo ink would induce oxidative stress in humans. One possible explanation of this paradoxical finding is related to chemical variability of color tattoo inks briefly discussed in the introduction. A tattoo in this particular experiment was blue (Fig 1A) and most of the modern blue tattoo inks are based on copper(II) phthalocyanine (CuPC) also known as Phthalocyanine Blue BN, a bright crystalline synthetic blue pigment first prepared by accident in 1927 (Lobbert 2000) and extensively studied in industrial context due to its interesting physicochemical properties. For example, CuPC is a well suited material for organic solar cells (Ali et al. 2020) and it has been suggested for data storage in quantum computing (Warner et al. 2013). Considering its low toxicity (eg. FDA approval for use in infant furniture and toys, and contact lenses (Athar and Farrukh 2019; PubChem)) and compelling properties, CuPC might even turn out to be interesting in the growing field of biocompatible organic bioelectronics (Feron et al. 2018). Although biological effects of CuPC are yet to be elucidated, one especially interesting finding relevant for this paper is related to its strong antioxidant activity explored for the first time in 2012 by Amaral et al. where they showed that phthalocyanines were able to both reduce and prevent lipid peroxidation in mouse brain, 
kidney and liver (Amaral et al. 2012). Moreover, CuPC (alongside manganese(II) phthalocyanine was found to be especially protective in this study as it also exerted strong antioxidant properties in $\mathrm{H}_{2} \mathrm{O}_{2}, \mathrm{Fe}^{2+}$ and $\mathrm{H}_{2} \mathrm{O}_{2}+\mathrm{Fe}^{2+}$-induced oxidative stress in deoxyribose degradation assay (Amaral et al. 2012). Based on these results authors suggest that CuPC might act by inhibiting generation of free radicals, or inhibiting their actions against lipids. This is in concordance with the results of this study as overall RRN of the tattoo was shifted towards antioxidative values (Fig 1C, Fig 1E, Fig 1F), and the protective effect was especially pronounced for lipid peroxidation (Fig 1I, Table 1). Authors also suggest that phthalocyanines might exert their antioxidant properties by directly degrading hydroperoxides such as $\mathrm{H}_{2} \mathrm{O}_{2}$. This is very interesting as the second most pronounced change of RRN subsystems observed in this study was related to sample $\mathrm{H}_{2} \mathrm{O}_{2}$ dissociation rate $(+11.8 \%$ vs CTR). I originally assumed that the effect might be related to modulation of endogenous peroxidases, enzymes that play one of the most important roles in cellular defense against oxidative stress, however it is possible that the change in the $\mathrm{H}_{2} \mathrm{O}_{2}$ dissociation rate was also mediated by CuPC present in the tattoo sample (Fig 2). Even though CuPC is the most prominent chemical constituent of blue tattoo inks, other chemicals or possibly even contaminants might be responsible for the observed effect and further research is needed to answer the intriguing question of how tattooing might shift local skin redox balance towards antioxidative values and reduce lipid peroxidation. Another possibility is the hormetic effect of tattoo ink as small quantities of harmful oxidative substances might trigger hypercompensation and induce net increase of the total antioxidant capacity (Homolak et al. 2020b). This interesting phenomena has been observed for 3,5,4'-trihydroxy-trans-stilbene (resveratrol), a well known wine polyphenol with myriad of health benefits exerted through potentiation of the antioxidant systems (Plauth et al. 2016).

Apart from the exact mechanism that might be responsible for the apparent paradoxical reductive potential of the tattoo, numerous other questions remain to be answered. For example, as the experiment was conducted during the spring, and the tattoo has not been exposed to the sun for several hours prior to testing, it would be interesting to see whether the observed effect would also be present after it was exposed to UV light as it has been shown that polycyclic aromatic hydrocarbons and other components of tattoo ink can generate ROS upon exposure to solar radiation (Regensburger et al. 2010), and sun-induced tattoo-related complaints are a common finding among inked people. Considering the fact that CuPC is popular in material sciences due to its photovoltaic effect, the ability to generate electric current upon exposure to light, it is possible that the same effect in the human body is responsible for the oxidative shift of the $R R N$ upon exposure to solar radiation and possibly related to unpleasant sensations such as itching. However, the author never experienced this so far. Another interesting question is related to the fact that due to the apparent paucity of data, the explanation of the observed effects with the color of the tattoo is highly hypothetical, and it is possible that other tattoos might also demonstrate antioxidant properties.

\section{Limitations of the study}

The study presented here is obviously limited by its nature because it is an N-of-1 study. Although numerous control procedures were introduced to maximally reduce experimental error (eg. technical replicates, multiple measure points, carefully chosen prick-sampling anatomical site, scrupulous setup of biochemical experiments), none of the precautionary settings can overcome a limitation that the presented results reflect a single person, a single time-point and a single tattoo. It would also be fair to point out that the author of the paper was 
at the same time the only subject in the experiments so this should also be taken into account. However, this didn't affect the experiment or writing of this manuscript in any way.

\section{Conclusion:}

In conclusion, this $\mathrm{N}$-of- 1 study provides the first evidence of the effect of a tattoo on the human redox regulatory network and oxidative stress parameters. Furthermore, it provides preliminary evidence that there is a possibility of antioxidative, in contrast to the expected pro oxidative changes following the administration of tattoo ink into the skin, and raises the question of different biological effects that might be mediated by a variety of chemical constituents of coloured inks.

\section{Data availability statement}

All data generated by this research has been presented in the manuscript. Raw data can be obtained from the author's GitHub account: https://github.com/janhomolak

\section{References:}

Ali AM, Said DA, Khayyat M, Boustimi M, Seoudi R. Improving the efficiency of the organic solar cell (CuPc/C60) via PEDOT: PSS as a photoconductor layer doped by silver nanoparticles. Results in Physics. 2020;16:102819 Available from: https://linkinghub.elsevier.com/retrieve/pii/S2211379719325495

Amaral GP, Puntel GO, Dalla Corte CL, Dobrachinski F, Barcelos RP, Bastos LL, et al. The antioxidant properties of different phthalocyanines. Toxicol. In Vitro. 2012;26(1):125-32 Available from: http://dx.doi.org/10.1016/j.tiv.2011.10.006

Arl M, Nogueira DJ, Schveitzer Köerich J, Mottim Justino N, Schulz Vicentini D, Gerson Matias W. Tattoo inks: Characterization and in vivo and in vitro toxicological evaluation. J. Hazard. Mater. 2019;364:548-61 Available from: http://dx.doi.org/10.1016/j.jhazmat.2018.10.072

Athar M, Farrukh S. Article on undesirable effects of different chemicals used in tattoo inks. MOJ Toxicology. MedCrave Publishing; 2019;5(2):62-5 Available from:

https://medcraveonline.com/MOJT/MOJT-05-00154.php

Bäumler W, Eibler ET, Hohenleutner U, Sens B, Sauer J, Landthaler M. Q-switch laser and tattoo pigments: first results of the chemical and photophysical analysis of 41 compounds. Lasers Surg. Med. 2000;26(1):13-21 Available from: 3.0.co;2-

s">http://dx.doi.org/10.1002/(sici)1096-9101(2000)26:1<13::aid-Ism4>3.0.co;2-s

Cao T, He M, Bai T, Liu H. Establishment of a Method for Measuring Antioxidant Capacity in Urine, Based on Oxidation Reduction Potential and Redox Couple I/KI. Bioinorg. Chem. Appl. 2016;2016:7054049 Available from: http://dx.doi.org/10.1155/2016/7054049

Falconi M, Teti G, Zago M, Galanzi A, Breschi L, Pelotti S, et al. Influence of a commercial tattoo ink on protein production in human fibroblasts. Arch. Dermatol. Res. 2009;301(7):539_ 47 Available from: http://dx.doi.org/10.1007/s00403-009-0953-7

Feron K, Lim R, Sherwood C, Keynes A, Brichta A, Dastoor PC. Organic Bioelectronics: Materials and Biocompatibility. Int. J. Mol. Sci. 2018;19(8) Available from: 
http://dx.doi.org/10.3390/ijms19082382

Forte G, Petrucci F, Cristaudo A, Bocca B. Market survey on toxic metals contained in tattoo inks. Sci. Total Environ. 2009a;407(23):5997-6002 Available from:

http://dx.doi.org/10.1016/j.scitotenv.2009.08.034

Forte G, Petrucci F, Cristaudo A, Bocca B. Quantification of Sensitizing Metals in Tattooing Pigments by SF-ICP-MS Technique. TOCBMJ. 2009b;2(2):42-7 Available from:

http://www.bentham-

open.org/pages/content.php?TOCBMJ/2009/00000002/00000002/42TOCBMJ.SGM

Hadwan $\mathrm{MH}$. Simple spectrophotometric assay for measuring catalase activity in biological tissues. BMC Biochem. 2018;19(1):7 Available from: http://dx.doi.org/10.1186/s12858-0180097-5

Homolak J, Kodvanj I, Babic Perhoc A, Virag D, Knezovic A, Osmanovic Barilar J, Riederer $P$, Salkovic-Petrisic M. Nitrocellulose redox permanganometry: a simple method for reductive capacity assessment bioRxiv 2020.06.16.154682; doi: 10.1101/2020.06.16.154682

Homolak J, Babic Perhoc A, Knezovic A, Kodvanj I, Virag D, Osmanovic Barilar J, Riederer $P$, Salkovic-Petrisic M. Activation of redox regulatory network and disinhibition of the hippocampal protective oxidative pentose phosphate pathway: a possible explanation of the hormetic effects of galactose [manuscript in preparation] 2020.

Høgsberg T, Jacobsen NR, Clausen PA, Serup J. Black tattoo inks induce reactive oxygen species production correlating with aggregation of pigment nanoparticles and product brand but not with the polycyclic aromatic hydrocarbon content. Exp. Dermatol. 2013;22(7):464-9 Available from: http://dx.doi.org/10.1111/exd.12178

Hutton Carlsen K, Serup J. Photosensitivity and photodynamic events in black, red and blue tattoos are common: A "Beach Study." J. Eur. Acad. Dermatol. Venereol. 2014;28(2):231-7 Available from: http://dx.doi.org/10.1111/jdv.12093

Laux P, Tralau T, Tentschert J, Blume A, Dahouk SA, Bäumler W, et al. A medicaltoxicological view of tattooing. Lancet. 2016;387(10016):395-402 Available from: https://linkinghub.elsevier.com/retrieve/pii/S014067361560215X

Li X. Improved pyrogallol autoxidation method: a reliable and cheap superoxide-scavenging assay suitable for all antioxidants. J. Agric. Food Chem. 2012;60(25):6418-24 Available from: http://dx.doi.org/10.1021/jf204970r

Lobbert G, editor. Phthalocyanines. Ullmann's Encyclopedia of Industrial Chemistry. Weinheim, Germany: Wiley-VCH Verlag GmbH \& Co. KGaA; 2000. p. 241 Available from: http://doi.wiley.com/10.1002/14356007.a20_213

Neale PA, Stalter D, Tang JYM, Escher BI. Bioanalytical evidence that chemicals in tattoo ink can induce adaptive stress responses. J. Hazard. Mater. 2015;296:192-200 Available from: http://dx.doi.org/10.1016/j.jhazmat.2015.04.051

Plauth A, Geikowski A, Cichon S, Wowro SJ, Liedgens L, Rousseau M, et al. Hormetic shifting of redox environment by pro-oxidative resveratrol protects cells against stress. Free Radic. Biol. Med. 2016;99:608-22 Available from: http://dx.doi.org/10.1016/j.freeradbiomed.2016.08.006

Prabhakar PV, Reddy UA, Singh SP, Balasubramanyam A, Rahman MF, Indu Kumari S, et al. Oxidative stress induced by aluminum oxide nanomaterials after acute oral treatment in 
Wistar rats. J. Appl. Toxicol. 2012;32(6):436-45 Available from: http://dx.doi.org/10.1002/jat.1775

PubChem. Copper(II) phthalocyanine [Internet]. PubChem. [cited 2020 May 25]. Available from: https://pubchem.ncbi.nlm.nih.gov/compound/8978

Regensburger J, Lehner K, Maisch T, Vasold R, Santarelli F, Engel E, et al. Tattoo inks contain polycyclic aromatic hydrocarbons that additionally generate deleterious singlet oxygen. Exp. Dermatol. 2010;19(8):e275-81 Available from: http://dx.doi.org/10.1111/j.16000625.2010.01068.x

Serup J, Hutton Carlsen K, Dommershausen N, Sepehri M, Hesse B, Seim C, et al. Identification of pigments related to allergic tattoo reactions in 104 human skin biopsies. Contact Dermatitis. 2020;82(2):73-82 Available from: http://dx.doi.org/10.1111/cod.13423

Srivastav AK, Dubey D, Chopra D, Singh J, Negi S, Mujtaba SF, et al. Oxidative stressmediated photoactivation of carbazole inhibits human skin cell physiology. J. Cell. Biochem. 2020;121(2):1273-82 Available from: http://dx.doi.org/10.1002/jcb.29360

Van der Plancken I, Van Loey A, Hendrickx MEG. Changes in sulfhydryl content of egg white proteins due to heat and pressure treatment. J. Agric. Food Chem. 2005;53(14):572633 Available from: http://dx.doi.org/10.1021/jf050289+

Warner M, Din S, Tupitsyn IS, Morley GW, Stoneham AM, Gardener JA, et al. Potential for spin-based information processing in a thin-film molecular semiconductor. Nature.

2013;503(7477):504-8 Available from: http://dx.doi.org/10.1038/nature12597 
A

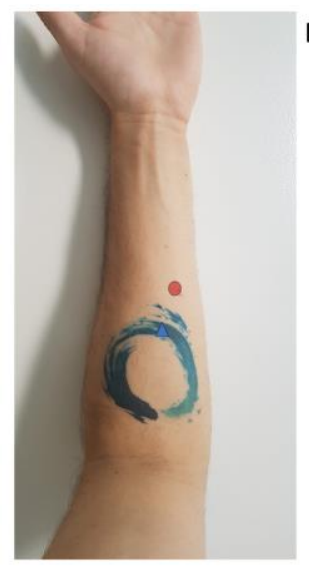

D

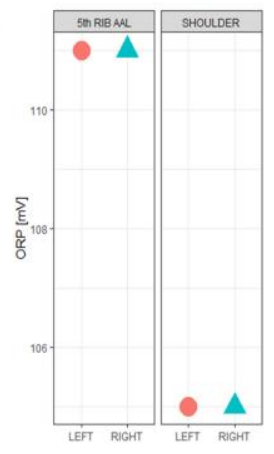

G

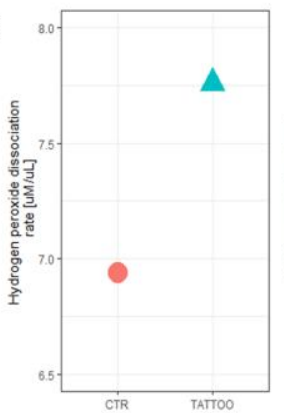

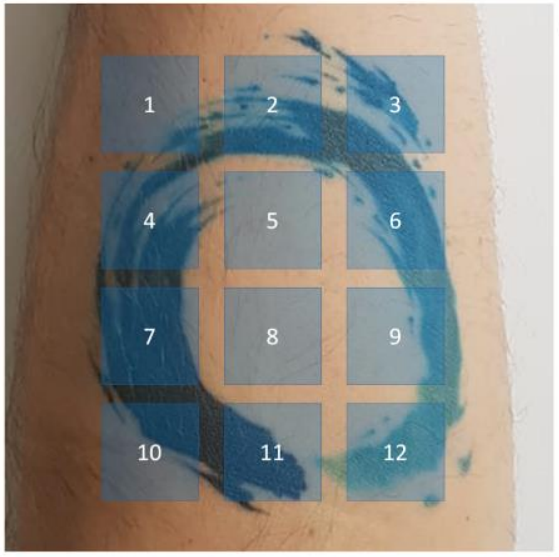

C

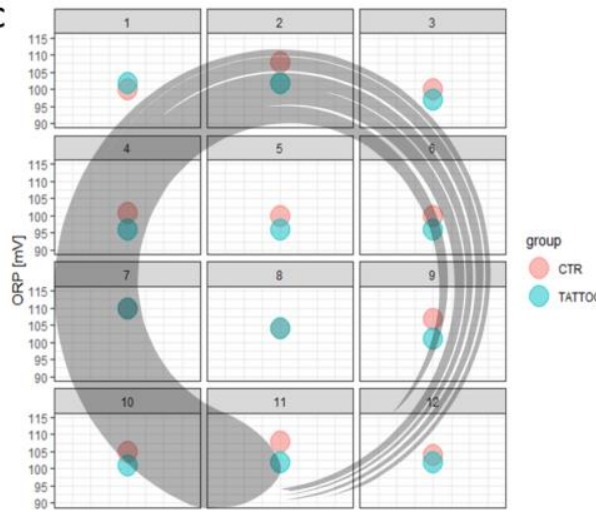

E

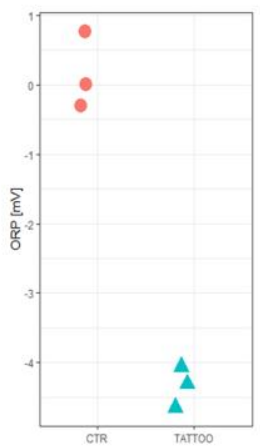

F

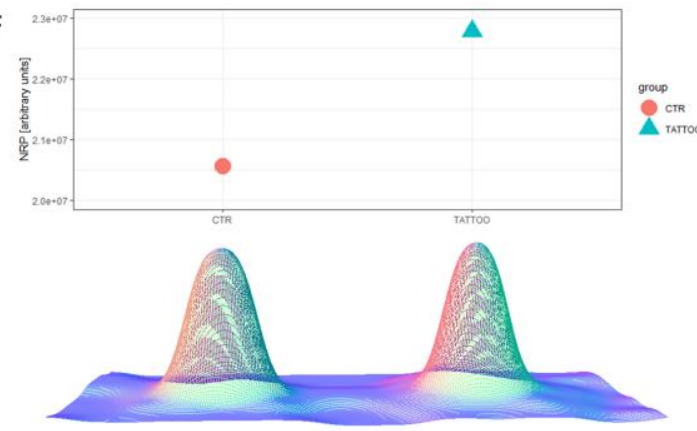

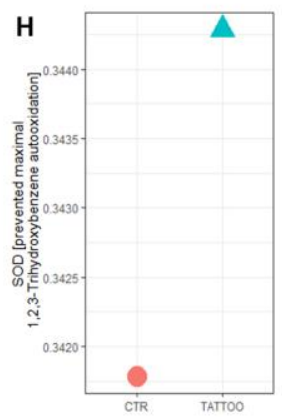
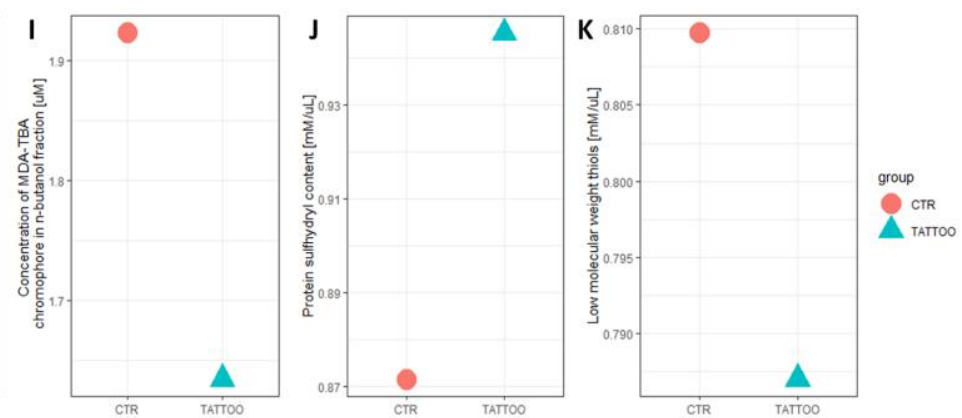

Fig 1. A blue tattoo redox regulatory network analysis. A) A photograph of the tattoo on the left forearm with the area used for prick-sampling for the tattoo sample indicated with a blue triangle, and the area used for prick-sampling of the control sample indicated with a red circle. B) Enlarged photograph of the tattoo with 12 sampling areas used for ORP measurements illustrated with blue squares. The same anatomical area on the other forearm was used for control measurements. C) Results of the ORP analysis with potentials from the tattooed forearm depicted with blue circles, and potentials from the corresponding areas on the right forearm presented with red circles. An illustration of the tattoo is overlaid onto the graph. D) Control measurements of ORP to control for possible ipsilateral-contralateral differences in reductive potential. Two control areas are illustrated - an area above the fifth rib in the anterior axillary line $\left(5^{\text {th }}\right.$ RIB AAL) and clavicular (anterior) part of the deltoid muscle (SHOULDER). E) Redox regulatory network subsystem analysis in the sample obtained from tattooed and 
control area by prick-sampling method as illustrated in Fig 1A. Three technical replicates of normalized oxidation-reduction potential measurements of $\mathrm{I}_{2} / \mathrm{KI}$-redoxstabilized samples. F) Nitrocellulose Redox Permanganometry of the samples presented as Gel Analyzer-based densitometric quantification (upper) and 3D gradient surface plot (lower). G) Hydrogen peroxide dissociation rate assessed by quantification of carbonato-cobaltate(III) complex $\left.\left(\left[\mathrm{Co}\left(\mathrm{CO}_{3}\right)_{3}\right] \mathrm{Co}\right) \mathrm{H}\right)$ Activity of superoxide dismutase measured by 1,2,3-Trihydroxybenzene autooxidation inhibition I) Lipid peroxidation quantified by thiobarbituric acid reactive substances assay J) Protein sulfhydryl content assessed by 5-thio-2-nitrobenzoic acid (TNB) quantification in protein precipitate K) Low molecular weight thiol concentration assessed by 5-thio-2nitrobenzoic acid (TNB) quantification in sample supernatant. 


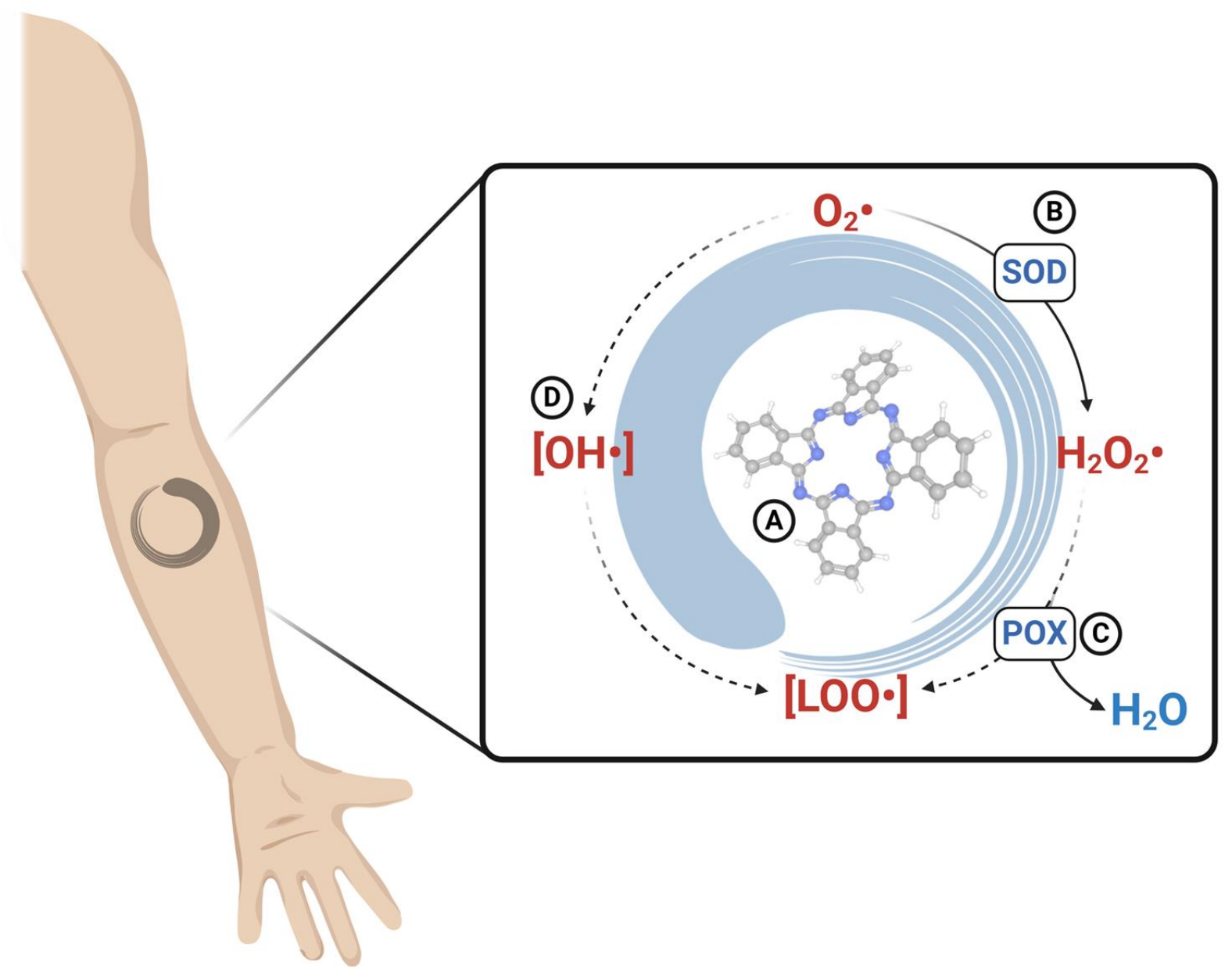

Fig 2. A schematic representation of the antioxidative effect of blue tattoo ink possibly mediated by copper(II) phthalocyanine (CuPC) and correspondent observed effects. A) Chemical structure of the CuPC. B) Slight activation of the superoxide dismutase with consequent detoxification of superoxide radicals to hydrogen peroxide $\left(\mathrm{H}_{2} \mathrm{O}_{2}\right)$. C) Activation of endogenous peroxidases or possible direct catalytic activity towards hydrogen peroxide transforms harmful $\mathrm{H}_{2} \mathrm{O}_{2}$ into water and inhibits peroxide-mediated potentiation of lipid peroxidation. D) Inhibition of the lipid peroxidation system through the unknown mechanism. SOD - superoxide dismutase; POX - peroxidase. 
Table 1. Differences between redox regulatory network subsystem parameters between the tattoo and the control sample. Usual interpretation of the oxidative-stress-related changes of redox regulatory network subsystems is presented in column "Oxidative stress" with changes indicative of reduced oxidative stress in a tattoo sample marked with an arrow facing downward $(\downarrow)$ and highlighted in green, and changes indicative of increased oxidative stress marked with an arrow facing upward ( $\uparrow)$ and highlighted in red.

\begin{tabular}{|l|l|c|c|}
\hline Redox regulatory network subsystem & \multicolumn{1}{|c|}{ Assessment method } & $\begin{array}{c}\text { Change vs. } \\
\text { CTR }\end{array}$ & $\begin{array}{c}\text { Oxidative } \\
\text { stress }\end{array}$ \\
\hline \multirow{2}{*}{ Overall reductive capacity } & I2/KI-redox-stabilized oxidation-reduction potential (ORP) & $-4.33 \mathrm{mV}$ & $\downarrow$ \\
\hline & Nitrocellulose Redox Permanganometry (NRP) & $+1,1 \%$ & $\downarrow$ \\
\hline Catalase/Peroxidase activity & $\mathrm{H}_{2} \mathrm{O}_{2}$ dissociation rate & $+11.8 \%$ & $\downarrow$ \\
\hline Superoxide dismutase activity & Inhibition of 1,2,3-Trihydroxybenzene autooxidation & $+0.7 \%$ & $\downarrow$ \\
\hline Lipid peroxidation & Quantification of MDA-TBA chromophore & $-15.0 \%$ & $\downarrow$ \\
\hline Protein sulfhydryl content & Quantification of 5-thio-2-nitrobenzoic acid (TNB) & $+8.5 \%$ & $\downarrow$ \\
\hline Low molecular weight thiols & Quantification of 5-thio-2-nitrobenzoic acid (TNB) & $-2.8 \%$ & $\uparrow$ \\
\hline
\end{tabular}

